\title{
Essais
}

Revue interdisciplinaire d'Humanités

\section{De la prothèse réparatrice au corps amplifié}

\section{Aurélie Martinez}

\section{OpenEdition}

Journals

Édition électronique

URL : https://journals.openedition.org/essais/10375

DOI : 10.4000/essais. 10375

ISSN : 2276-0970

\section{Éditeur}

École doctorale Montaigne Humanités

\section{Édition imprimée}

Date de publication : 15 novembre 2012

Pagination : 45-51

ISBN : 978-2-86781-857-8

ISSN : 2417-4211

Référence électronique

Aurélie Martinez, «De la prothèse réparatrice au corps amplifié », Essais [En ligne], 2 | 2012, mis en ligne le 17 janvier 2022, consulté le 20 janvier 2022. URL : http://journals.openedition.org/essais/ 10375 ; DOI : https://doi.org/10.4000/essais.10375 
Aurélie Martinez, Laboratoire CLARE/Artes, Université Michelde-Montaigne Bordeaux 3, aureliemartinez23@gmail.com

\section{Résumé}

Selon la pensée mécaniste de René Descartes, le corps humain est comparable à une machine. Si l'un de ces composants est endommagé, sa dite normalité devient pathologique. Pour rétablir ces fonctions corporelles, les scientifiques proposent à leurs patients des prothèses. Dans l'art actuel, des artistes tels que Matthew Barney, Joël-Peter Witkin et Stelarc mettent en scène ces individus prothétiques ou s'en inspirent en travaillant sur un corps amplifié ou prolongé.

\section{Mots-clés}

Corps, chair, prothèse, corps amplifié, art contemporain.

\section{Abstract}

From Functional Prosthesis to the Extended Body

According to the mechanistic system of René Descartes, the human body is like a machine. If one of his components is damaged, its standard condition becomes pathological. To restore those dysfunctions, scientists can provide prosthesis to their patients. In art today, artists such as Matthew Barney, Joël-Peter Witkin and Stelarc create and stage those prosthetic individuals or get inspired by working on an extended body.

\section{Keywords}

Body, flesh, prosthesis, extended body, contemporary art. 


\section{De la prothèse réparatrice au corps amplifié}

\section{Aurélie Martinez}

L'organisation du corps humain est comparable à celle d'une machine. Le fonctionnement de celle-ci dépend de la bonne coordination d'organes interdépendants, semblables à des moteurs composés de rouages, de ressorts, de courroies, de gaines, de pistons, etc. Lors d'un accident, suite à une maladie ou à une déficience congénitale, la machine humaine peut être endommagée d'une manière interne ou externe. Mais à quel moment doit-on considérer que la dite normalité du corps bascule vers un état pathologique irréversible? Pour distinguer le rapport entre un état normal et un état pathologique, une référence à la définition de la normalité énoncée par Georges Canguilhem, dans la seconde partie de son ouvrage Le Normal et le pathologique, semble nécessaire:

Est normal, étymologiquement, puisque norma, ce qui désigne l'équerre, ce qui ne penche ni à droite ni à gauche, donc ce qui se tient dans un juste milieu, d'où deux sens dérivés: est normal ce qui est tel qu'il doit être: est normal, au sens le plus usuel du mot, ce qui se rencontre dans la majorité des cas d'une espèce déterminée ou ce qui constitue soit la moyenne soit le module d'un caractère mesurable. ${ }^{1}$

Cette définition extraite des préceptes de la philosophe réaliste indique que tout être humain possède un corps parfait du moment où il maintient au mieux ses fonctions vitales. Chaque jour, l'homme mange, boit, digère, défèque, urine, marche, dort, court, fait plus ou moins du sport, etc. Toutes ces actions liées au bon fonctionnement du corps sont semblables à une thérapie naturelle. Si le corps est mal alimenté, s'il ne se repose pas correctement, s'il ne se déplace pas selon ses besoins journaliers, etc., la thérapie qui entretient le mécanisme naturel de notre organisme se détériore ou se dérègle. La normalité corporelle devient pathologique lorsque le patient n'est plus à même de procéder à sa thérapie quotidienne qui entretient naturellement son corps. En cas de pathologie grave, la santé physique peut basculer d'un état stable

1. Georges Canguilhem, Le Normal et le pathologique (1966), Paris, Presses Universitaires de France, 2006, p. 76. 
vers une anormalité passagère soignable au moyen d'une thérapie médicale. Dans d'autres cas plus sévères, le diagnostic ne peut qu'annoncer un handicap irréversible menaçant ou empêchant le rétablissement de l'état physique originel. Afin de compenser les fonctions corporelles défaillantes, les médecins peuvent proposer à leurs patients des prothèses.

\section{L'homme prothétique}

La définition du mot prothèse, venant du grec prostithenai, "ajouter à ", est à considérer selon deux degrés d'appréhension. Selon sa définition la plus courante, une prothèse désigne un appareillage orthopédique ou un dispositif implanté à l'intérieur de l'organisme pour suppléer à un organe manquant ou à une fonction compromise. Néanmoins, une prothèse peut aussi définir un objet ou une machine que nous utilisons au quotidien, afin d'améliorer ou de prolonger nos capacités physiques. La prolifération d'objets technologiques permettant à l'homme de rectifier, de combler ou d'augmenter certaines de ses capacités corporelles n’a jamais cessé de progresser. En 1929, Sigmund Freud en faisait déjà l'inventaire, dans le Malaise dans la civilisation.

Grâce à tous ces instruments, l'homme perfectionne ses organes moteurs aussi bien que sensoriels [...]. Les machines à moteur le munissent de forces gigantesques aussi faciles à diriger à son gré que celles de ses muscles; grâce au navire, ni l'eau ni l'air ne peuvent entraver ses déplacements. Avec les lunettes, il corrige les défauts des lentilles de ses yeux; le télescope lui permet de voir à d'immenses distances, et le microscope de dépasser les limites étroites assignées à sa vision par structure de la rétine [...]. À l'aide du téléphone, il entend loin, à des distances que les contes eux-mêmes respecteraient comme infranchissables [...]. L'homme est devenu pour ainsi dire une sorte de dieu prothétique, dieu certes sill revêt tous ses organes auxiliaires...

De nos jours, ainsi que le fait remarquer Bernard Andrieu, dans son ouvrage intitulé Devenir hybride, certains de ces objets, qu'il qualifie de corporels, se sont peu à peu incorporés « en hybridant la chair par la technique: le Bluetooth dans l'oreille, le SMS au bout des doigts, le portable porté devant soi et la télécommande agissant sur mon corps par procuration ${ }^{2} »$. Par conséquent, bien qu'il existe une différence entre les objets technologiques que nous utilisons au quotidien et les prothèses réparatrices, les premiers sont en train de s'incorporer à notre chair ou tout au moins de s'en approcher au plus près.

Mais aujourd'hui, la prothèse qui nous intéresse est la prothèse thérapeutique.

2. Bernard Andrieu, Devenir hybride, Presses Universitaires de Nancy, 2008, p. 68-69. 


\section{La prothèse réparatrice}

La prothèse réparatrice, permettant à l'homme de pallier un handicap, le rend mi-organique, mi-prothétique. Ces prothèses ont considérablement évolué et permettent de soigner une multitude de pathologies. Elles comblent des déficiences du cristallin, de l'audition, des articulations, des dents, du crâne, du larynx, du cœur, des bras, des jambes, de la hanche, des genoux, etc. D'un point de vue historique, la première main artificielle et une emboîture engainant l'avant-bras, datant de 4000 ans av. J.-C., ont été retrouvées sur une momie égyptienne. Au III $^{\mathrm{e}}$ siècle av. J.-C., Serjius Silus, amputé de la main droite, se fit faire une main de fer. La jambe de bois de Capoue est doublée d'une gaine de bronze. Au Xv siècle, Léonard de Vinci dessinait un oil artificiel pendant que les horlogers suisses fabriquaient des mains articulées en fer forgé. Sur les champs de bataille, Ambroise Paré, chirurgien sous quatre rois, ampute, cautérise et remplace par des prothèses fonctionnelles les membres meurtris. La prothèse demeure une chirurgie réparatrice plutôt qu'esthétique qui doit restaurer l'image corporelle de l'amputé de la guerre, comme celui de la Première Guerre mondiales.

Depuis, l'apparition de la "bionique humaine» a permis d'immenses progrès. Claudia Mitchell, amputée du bras gauche au ras de l'épaule en 2004, suite à un accident de moto, a pu bénéficier en août 2005, comme cinq autres sujets, d'une méthode expérimentale révolutionnaire d'installation d'une prothèse totale de bras mise au point par les chirurgiens et chercheurs du Rehabilitation Institute of Chicago (Illinois). Le cas de Claudia Mitchell a ouvert une nouvelle ère de l'ingénierie bionique: les chirurgiens sont en effet parvenus à reconnecter indirectement son cerveau avec son bras prothétique. Comment? En utilisant son anatomie. Les extrémités des nerfs moteurs coupés du bras amputé (nerf médian, nerf radial, nerf brachial) ont été déroutées chirurgicalement vers un groupe de muscles de la paroi thoracique. Le circuit moteur normal, qui part du cortex moteur du cerveau, descend dans la moelle épinière et sort entre les vertèbres pour constituer les nerfs périphériques, a été conservé. Commandés par un circuit moteur cérébral, les muscles thoraciques se contractent et envoient à travers la peau des signaux myoélectriques ${ }^{4}$. Ces messages sont relayés par plusieurs dizaines de capteurs collés sur la poitrine vers la prothèse pour la mettre en mouvement. L'extrémité du nerf médian amputé est par exemple connectée au muscle pectoral, qui envoie un signal "ouvert/fermé " à la pince des doigts. La puce est capable d'analyser une centaine de signaux neuronaux et de commander jusqu'à 22 fonctions distinctes de la prothèse.

3. Ibid., p. 69-70.

4. Qui se rapporte à la propriété électrique des muscles. 
Ces êtres prothétiques dont le corps s'est hybridé pour retrouver une meilleure fonctionnalité attirent l'attention des artistes contemporains. Certains les mettent en scène dans leurs œuvres en détournant la fonction première de leurs prothèses. D'autres, investis dans l'évolution constante des recherches en cybernétique, agrémentent leur corps pour mieux le prolonger et l'amplifier. Comment doit-on appréhender l'exploitation de ces technologies dans l'art actuel?

Aimee Mullins, née avec une hémimélie fibulaire (c'est-à-dire sans péroné) a été amputée sous le genou au cours de sa première année. Depuis, elle est devenue athlète para-olympique, mannequin et actrice dans les films de l'artiste Matthew Barney. Pour Matthew Barney, le handicap d'Aimee Mullins est une source d'inspiration. Pour chacun de ses rôles, Matthew Barney lui crée une nouvelle paire de jambes. Chacune d'entre elles lui donne une identité bien particulière. Dans Cremaster 3, elle incarne l'alter ego de l'Apprenti. Ses jambes semblent être faites de cristal afin d'amplifier la pureté que son personnage dégage. Suite à une transformation fortuite liée au déroulement du film, elle devient une femme-léopard. Ses prothèses deviennent des pattes. En 1992, dans une de ses photographies, intitulée Satiro, Joël-Peter Witkin affuble l'un de ses modèles, né sans bras et sans jambe, d'une paire de pattes de cervidé afin de lui donner l'identité d'un satyre. Face à ces êtres hybrides, nous constatons que le détournement de la prothèse thérapeutique permet de donner momentanément une nouvelle identité au corps porteur.

\section{Le corps amplifié}

Selon une tout autre approche, le performeur australien Stelarc, pionnier du body-art cybernétique, a développé une esthétique prothétique où "l'artiste est un guide dans l'évolution qui extrapole et imagine de nouvelles trajectoires $[\ldots]$, qui restructure et hypersensibilise le corps $s^{5} »$.

Equipé de son corps amplifié, de ses yeux lasers, de sa troisième main, de son bras automatique et de son ombre vidéo, [Stelarc] incarne à l'avance l'bybride humain-machine que nous sommes tous symboliquement en train de devenir. ${ }^{6}$

Pour lui, "plutôt que de remplacer une partie du corps qui manque ou qui fonctionne mal, ces interfaces et ces dispositifs augmentent ou amplifient la forme et les fonctions du corps ${ }^{7} »$. Selon son mode de pensée, le corps

5. Mark Dery, Vitesse virtuelle, La cyberculture aujourd'hui (1996), Paris, éditions Abbeville, 1997, p. 166.

6. Ibid.

7. Bernard Andrieu, Devenir hybride, op. cit., p. 70. 
organique est obsolète. Ses recherches sont à l'opposé « du retour à l'organique pur dont peuvent faire état des œuvres telles que la robe ou la veste de viande dont se recouvrent respectivement, au même moment, Jana Sterbak ou Robert Gligorov, signe d'un désir de renouement brut avec la matière vivante ${ }^{8}$ ». Pour Stelarc, "l'humain prolongé, c'est celui qui use de prothèses efficaces, qui démultiplient ses pouvoirs sensibles par l'adjonction à sa propre structure d'éléments corporels" ". Avec "Writing with Three Hands Simultaneously (1981) ou encore The Third Arm (1995), l'artiste ajoute à son corps un bras articulé mécanique ou une troisième main lui permettant d'augmenter d'un tiers la force de ses membres supérieurs ou de ses capacités de préhension manuelles ${ }^{10}$ ".

Fabriquée sur mesure par un fabricant japonais, la Main est un manipulateur robotique d'une grande dextérité, qui peut être commandé par les signaux EMG (électromyographiques) émis par les muscles du ventre et des cuisses de Stelarc. Elle peut pincer, saisir, lâcher, tourner son poignet à deux cent quatre-vingt-dix degrés dans les deux directions, et possède un système de feed-back ${ }^{11}$ tactile qui donne un sens rudimentaire du toucher en stimulant les électrodes fixées au bras de l'artiste. ${ }^{12}$

"Autre réalisation [...] une machine dotée de six jambes dont le fonctionnement est régulé par le pas d'un marcheur ${ }^{13}$ " nommée Exoskeleton qu'il présente en 1998. Composé de six pattes, cet impressionnant robot a été développé en collaboration avec des ingénieurs de Hambourg. Cet hybride mi-homme, mi-machine propulsé par un mécanisme à air comprimé, permet à l'homme de développer l'ensemble de ses mouvements. Debout en son centre, il le dirige en agitant ses bras. Les propositions de Stelarc semblent, sans conteste, valoriser " une culture délirante de l'augmentation corporelle nécessaire. [...] le corps seul est faible, ce n'est qu'une fois machiné qu'il devient son propre au-delà - corps que l'on va doter pour cela de supermembres dont la fonction, selon l'usage classique est de prolonger le naturel. [...] Le principe en résulte que le corps est dépassé [...] le statut biologique de l'humain, de toute façon, est appelé à varier, à muter sous la pression de la technique. Rien à cela de fatal, une telle évolution est logique, elle ne fait que prolonger les

8. Paul Ardenne, L'image corps. Figures de l'humain dans l'art du $\mathrm{xx}^{\mathrm{e}}$ siècle, Paris, Éditions du regard, 2001, p. 437.

9. Ibid., p. 438.

10. Ibid.

11. Rétroaction, action de contrôle en retour.

12. Mark Dery, Vitesse virtuelle, op. cit.

13. Ibid. 
évolutions déjà constatées par le passé, à commencer par l'exploitation de l'artificiel ${ }^{14}$ ».

Je conclurais donc par le questionnement suivant: que doit-on penser des androïdes mis au point par le roboticien japonais Hiroshi Ishiguro, directeur du Intelligent Robotics Laboratory, à l'Université d'Osaka, lorsque celui-ci présente à la presse internationale un androïde à son image, ou lorsqu'un metteur en scène japonais annonce la conception d'une pièce de théâtre dont l'actrice principale est une androïde nommée Geminoid $F$ ? Face à ces avancées en matière de robotique, une interrogation se profile: Le clone humain que l'on imagine aujourd'hui organique ne sera-t-il pas plutôt artificiel au même titre qu'un androïde?

\section{Bibliographie}

Andrieu Bernard, Devenir hybride, Nancy, Presses Universitaires de Nancy, 2008.

Ardenne Paul, L'image corps. Figures de l'humain dans l'art du $\mathrm{xx}^{\mathrm{e}}$ siècle, Paris, Éditions du regard, 2001.

Canguilhem Georges, Le normalet lepathologique, Paris, Presses Universitaires de France, 2006.

Dery Mark, Vitesse virtuelle, La cyberculture aujourd'hui [1996], Paris, éditions Abbeville, 1997.

Freud Sigmund, Le Malaise dans la civilisation [1929], Paris, Presses Universitaires de France, 2004.

14. Paul Ardenne, L'image corps, op. cit., p. 439. 